Meta

Journal des traducteurs

Translators' Journal

\title{
La liberté en traduction. Colloque international en hommage à D. Seleskovitch
}

\section{Georges L. Bastin}

Volume 36, numéro 2-3, juin 1991

URI : https://id.erudit.org/iderudit/001983ar

DOI : https://doi.org/10.7202/001983ar

Aller au sommaire du numéro

Éditeur(s)

Les Presses de l'Université de Montréal

ISSN

0026-0452 (imprimé)

1492-1421 (numérique)

Découvrir la revue

Citer cette note

Bastin, G. L. (1991). La liberté en traduction. Colloque international en

hommage à D. Seleskovitch. Meta, 36(2-3), 528-528.

https://doi.org/10.7202/001983ar d'utilisation que vous pouvez consulter en ligne. 


\section{BLOC-NOTES}

\section{LA LIBERTÉ EN TRADUCTION}

Colloque international en hommage à D. Seleskovitch

Les 7,8 et 9 juin 1990 s'est tenu à l'ESIT (Paris) un «vrai colloque»; «vrai», car il s'est agi d'un véritable débat autour d'un sujet commun traité par - seulement - 15 intervenants et quelque 280 participants. Au programme, quatre volets de l'activité traduisante.

\section{La traduction littéraire \\ (Président M. Gravier)}

Pour F. Israel (ESIT), la traduction littéraire est une appropriation du texte. L'essentiel n'est pas de reproduire mais de produire un texte (G. Toury). Pour ce faire, il fixe trois priorités: prééminence de la langue d'arrivée sur celle de départ, de l'effet sur le notionnel et de l'ensemble sur le détail. Après l'examen des causes d'appropriation (la nature du texte littéraire, la nécessaire intégration à la culture d'arrivée et la non-transparence du traducteur), il conclut qu'une fois respectée l'union de la lettre et du sens la traduction littéraire est une proposition personnelle dans un discours contraint: l'art du compromis.

R. Marsack (Prix Scott-Moncrief 1989) et C. Jardin ont, elles, livré des réflexions issues de leur expérience quotidienne, qui ont suscité un débat animé autour de plusieurs axes: l'attitude ancillaire du traducteur littéraire - les rapports auteur/traducteur - l'enseignement de cette spécialité - etc.

\section{La traduction technique}

(Président M. Pergnier et

\section{J. P. Van Deth)}

La communication de M. Cormier (Univ. de Montréal), la seule véritablement pédagogique et méthodologique, était un modèle du genre: justification et définition des objectifs généraux et spécifiques d'un cours de premier niveau, et une excellente description des étapes à faire parcourir à l'étudiant pour garantir une démarche traduisante réussie et créatrice. Communication exemplaire qui a néanmoins suscité de vives réserves quant à l'opportunité d'une décomposition aussi rigoureuse (qualifiée de «saucissonnage) du processus d'apprentissage.
Deux enseignantes de I'ESIT, F. Herbulot et C. Durieux, ont repris à leur compte toute l'expérience «européenne» (moins cloisonnée) de la traduction technique d'où ressort le besoin croissant de sortir des limites du texte pour atteindre l'acte de parole. La finalité prévaut toujours pour les textes pragmatiques. Retenons la métaphore du chat (circonspect, explorateur et colonisateur) et du pigeon voyageur (appropriateur et diffuseur) offerte par F. Herbulot pour cerner la tâche du traducteur technique. La liberté en traduction technique est synonyme de responsabilité et de créativité.

L'adaptation, évoquée à plusieurs reprises, a notamment été présentée comme une liberté, certes, mais aussi une fidélité à la «visée» de l'auteur, différente du sens mais non incompatible.

\section{L'interprétation de conférence}

\section{(Président R. Van Hoof-Haferkamp)}

Les interprètes ont avant tout parlé de contraintes (culturelles, linguistiques et diplomatiques) mais paradoxalement se sont affirmés dans une liberté dont les garants sont précisément les contraintes! Bien des questions ont aussi été soulevées par les interprètes auprès des tribunaux.

\section{La traductologie \\ (Président B. Pottier)}

Un échange nourri a marqué cette dernière séance, suscité moins par les communications du jour que par l'ensemble du colloque. À noter cependant l'exposé trop rapide de A. Hurtado; on gagnera à lire sa Fidélité en traduction (Didier Érudition, 1990). D. Seleskovitch, quant à elle, a souligné les liens entre pratique, recherche et enseignement. On en retiendra deux principes élémentaires mais non moins nécessaires: réintroduire l'homme dans le processus et découvrir des réalités non des abstractions.

$\mathrm{Ce}$ colloque «anti-marathon» (M. Lederer) a bien mis en évidence la filiation intime entre les différentes modalités de la traduction envers le vouloir-dire de l'auteur et envers le destinataire et sa langue; la liberté, quoi !

GeORGES L. BASTIN Caracas, Venezuela 